\title{
The utility of three different methods for measuring urinary 18-hydroxycortisol in the differential diagnosis of suspected primary hyperaldosteronism
}

\author{
R M Reynolds, L A Shakerdi ${ }^{1}$, K Sandhu ${ }^{2}$, A M Wallace ${ }^{1}$, P J Wood ${ }^{2}$ and B R Walker \\ Endocrinology Unit, School of Molecular \& Clinical Medicine, University of Edinburgh, Western General Hospital, Edinburgh EH4 2XU, UK, \\ ${ }^{1}$ Department of Clinical Biochemistry, Glasgow Royal Infirmary, Glasgow, UK and ${ }^{2}$ Regional Endocrine Unit, Southampton General Hospital, \\ Southampton UK
}

(Correspondence should be addressed to R M Reynolds; Email: r.reynolds@ed.ac.uk)

\begin{abstract}
Objective: Urine 18-hydroxycortisol (18-OHF) measurements are claimed to discriminate between primary hyperaldosteronism due to Conn's syndrome/adrenal adenoma or idiopathic bilateral adrenal hyperplasia (BAH), and also to identify cases of glucocorticoid-suppressible hyperaldosteronism (GSH). We have evaluated three urine 18-OHF methods using a panel of urine samples from patients with hypertension.

Design: Clinical methods comparative study.

Methods: Urine samples from patients with primary hyperaldosteronism due to either adenoma $(n=6)$, BAH $(n=6)$, GSH $(n=9)$, or essential hypertension $(n=38)$ were analysed without knowledge of the diagnosis using three different methods in different laboratories. These included 'in-house' radioimmunoassay (RIA), 'in-house' time-resolved fluorometric assay (DELFIA), and gas chromatography mass spectrometry (GC-MS).

Results: The three assays showed good correlation, but there were large bias differences: RIA bias was greater than DELFIA which was greater than GC-MS. Discrimination between adenoma and BAH patients was best for the DELFIA method, with no overlap between results for these two groups. All three methods gave significantly elevated results for the GSH group compared with the BAH and essential hypertension groups. No assay distinguished BAH from essential hypertension.

Conclusion: Measurement of urine 18-OHF may be a useful additional test in the differential diagnosis of primary hyperaldosteronism. The clinical diagnostic value of urinary 18-OHF measurements is method-dependent with the DELFIA assay having the best discriminatory value.
\end{abstract}

European Journal of Endocrinology 152 903-907

\section{Introduction}

Primary hyperaldosteronism is a potentially reversible cause of hypertension. Its prevalence amongst hypertensive patients is highly controversial, with estimates varying from affecting 5-13\% (1). The commonest causes are an aldosterone producing adenoma (Conn's syndrome) and idiopathic bilateral adrenal hyperplasia (BAH) with more rare causes including glucocorticoid suppressible hyperaldosteronism (GSH) and adrenal carcinoma. Establishing the cause is important to guide appropriate treatment as, for example, Conn's adenoma is potentially cured by surgery and GSH has the potential for remission of the disease with specific treatment using a synthetic glucocorticoid such as dexamethasone to suppress adrenocorticotropic hormone (ACTH). The standard methods to establish the differential diagnosis involve demonstrating suppression of plasma renin activity and investigating responsiveness of plasma aldosterone concentrations to variations in angiotensin II (e.g. in response to change in posture, angiotensin converting enzyme (ACE) inhibition, or diuretics) and ACTH (e.g. diurnal variation). Adrenal imaging is reserved for those with biochemically proven adrenal disease. These tests are disrupted by antihypertensive medication, complex to perform and interpret, and may not always be reliable (2).

Measurement of urine 18-hydroxycortisol (18-OHF) (11ß,17 $\alpha, 18,21$-tetrahydroxy-4-pregnene-3,20-dione) is claimed to be of value for the differential diagnosis of primary hyperaldosteronism $(3,4)$. 18-OHF is a hybrid steroid, secreted by the adrenal cortex from the action of aldosterone synthase (cytochrome P450 corticosterone methyl oxidase) on cortisol, rather than on its usual substrate corticosterone. Patients with Conn's adenoma or GSH reportedly excrete more of the steroid 
than patients with BAH (5). In Conn's adenoma, abnormal expression of steroidogenic enzymes in the tumour may explain over-production of $18-\mathrm{OHF}(6,7)$. In GSH an unequal translocation crossover results in a chimeric CYP11B1/CYP11B2 gene that has aldosterone synthase activity but is regulated by ACTH rather than angiotensin II $(8,9)$. The ectopic expression of the chimeric gene in the zona fasciculata exposes aldosterone synthase to abundant levels of cortisol not found in the zona glomerulosa.

To be of clinical use in the differential diagnosis of primary hyperaldosteronism, a simple method is required to measure $18-\mathrm{OHF}$ with high sensitivity and reproducibility. Here we report the evaluation of three different urine 18-OHF measurement methods using a panel of samples from patients under investigation for hypertension.

\section{Materials and methods}

We evaluated the measurement of $18-\mathrm{OHF}$ in urine samples from 70 patients, using three different methods in different laboratories. These included a radioimmunoassay (RIA), a time-resolved fluorometric assay (DELFIA), and gas chromatography mass spectrometry (GC-MS). Samples were analysed in random order without prior knowledge of the patient's clinical diagnosis. Of patients for whom notes were available $(n=59)$, diagnoses of Conn's adenoma $(n=6)$, GSH $(n=9)$, BAH $(n=6)$ and essential hypertension $(n=38)$ were established by a combination of the following: plasma renin activity and plasma aldosterone responses to posture and time, CT scanning, adrenal vein catheterisation, administration of dexamethasone and genotyping. All cases of adrenal adenoma were confirmed at surgery.

\section{Assays}

$\boldsymbol{R I A}$ 18-OHF was measured by direct RIA using a ${ }^{125} \mathrm{I}$ label as previously described (5). Briefly, samples were first incubated at room temperature with ${ }^{125} \mathrm{I}$ labelled 18-OHF-radioligand and rabbit 18-OHF antiserum (gifts from Professor C E Gomez-Sanchez, University of South Florida, Florida, USA) and then incubated overnight at $4{ }^{\circ} \mathrm{C}$ with donkey anti-rabbit serum and normal rabbit serum. Following centrifugation the supernatant was decanted and radioactivity counted in the remaining (antibody-bound) fraction using a Wallac Autogamma counter (Perkin-Elmer, Beaconsfield, Bucks, UK). The inter-assay coefficient of variation (CV) was $6.1-10.9 \%$ and intra-assay CV $8-22 \%$.

Delfia Microtitre strip plates (C12 'Maxisorp' plates, Nunc Ltd, Roskilde, Denmark) were coated overnight with goat anti-rabbit immunoglobulin (Dako Ltd, Ely, Cambridge, UK) and then washed three times. Following ether extraction and reconstitution in assay buffer, samples were incubated with biotinylated 18-OHF and rabbit anti-18-OHF antiserum (gifts from Professor C E Gomez-Sanchez, GV (Sonny) Montgomery Veterans Affairs Medical Center, MS, USA) for $1 \mathrm{~h}$ and washed three times. Europium-labelled Neutralite Avidin (prepared in-house) was added, incubated for $30 \mathrm{~min}$ and washed six times. Enhancement solution (PerkinElmer/Wallac) was added, mixed for $5 \mathrm{~min}$ and fluorescence read on an Arcus time-resolved fluorimeter. The inter-assay $\mathrm{CV}$ was $2.3-9.1 \%$ and intra-assay $\mathrm{CV}$ $2.2-8.6 \%$. Cross-reaction of the antiserum with 18 -hydroxycorticosterone was $0.18 \%$ and with cortisol was $0.08 \%$.

GC-MS Urinary steroid metabolites were measured by GC-MS using the methods of Shackleton (10) with minor modifications. Urinary steroids were extracted on Sep-Pak C18 cartridges and methoxime-trimethylsilyl derivatives formed using allo-tetrahydrodeoxycorticosterone as internal standard. 18-OHF was measured by GC-MS using GCQ Plus Benchtop Ion Trap GC/MS ${ }^{\mathrm{n}}$ (ThermoQuest, Finnigan, USA). The GC was fitted with a split/splitless injector operated in the splitless mode. The inlet temperature was held at $260{ }^{\circ} \mathrm{C}$ and the splitless time was $2 \mathrm{~min}$ at a constant septum purge. The right carrier was in constant pressure mode with an initial value of $40 \mathrm{kPa}$. The gas saver flow was $20 \mathrm{ml} / \mathrm{min}$ for $2 \mathrm{~min}$. Following injection, the temperature programme was as follows: $100{ }^{\circ} \mathrm{C}$ for $3 \mathrm{~min}, 20^{\circ} \mathrm{C} / \mathrm{minute}$ without hold for $7 \mathrm{~min}$ to $190^{\circ} \mathrm{C}, 2^{\circ} \mathrm{C} / \mathrm{minute}$ for $45 \mathrm{~min}$ to $285^{\circ} \mathrm{C}$. The temperature was held at $285^{\circ} \mathrm{C}$ for $10 \mathrm{~min}$, giving a total run time of $62 \mathrm{~min}$. The GC column (Liquid phase: DB-1. Dimensions: $30 \mathrm{~m} \times 0.322 \mathrm{~mm}$, film thickness $0.25 \mu \mathrm{m}$. J\&W Scientific, Folsom, CA, USA) was directly coupled, via a transfer line heated to $290^{\circ} \mathrm{C}$, to the ion source of the ion-trap mass spectrometer. Effluent steroids were fragmented and ionised by positive electron ionisation mode. This was interfaced to and controlled by a data system using GCQ XCalibur software (ThermoQuest Corporation, Austin, TX, USA) running under Windows NT. Upper limit of normal range $385 \mathrm{nmol} / 24 \mathrm{~h}$.

\section{Statistical analysis}

Differences between urine 18-OHF levels for each diagnosis for the three assays were compared by MannWhitney $U$ test. Correlation between assays was assessed by Spearman rank correlation and the three assays were compared by the Deming method comparison using Analyse-it (Leeds, UK).

\section{Results}

There was good correlation between assays (Delfia vs RIA, $r=0.72, \quad P<0.0001 ;$ GC-MS vs Delfia $r=0.64, \quad P<0.0001 ;$ GC-MS vs RIA, $r=0.49$, 

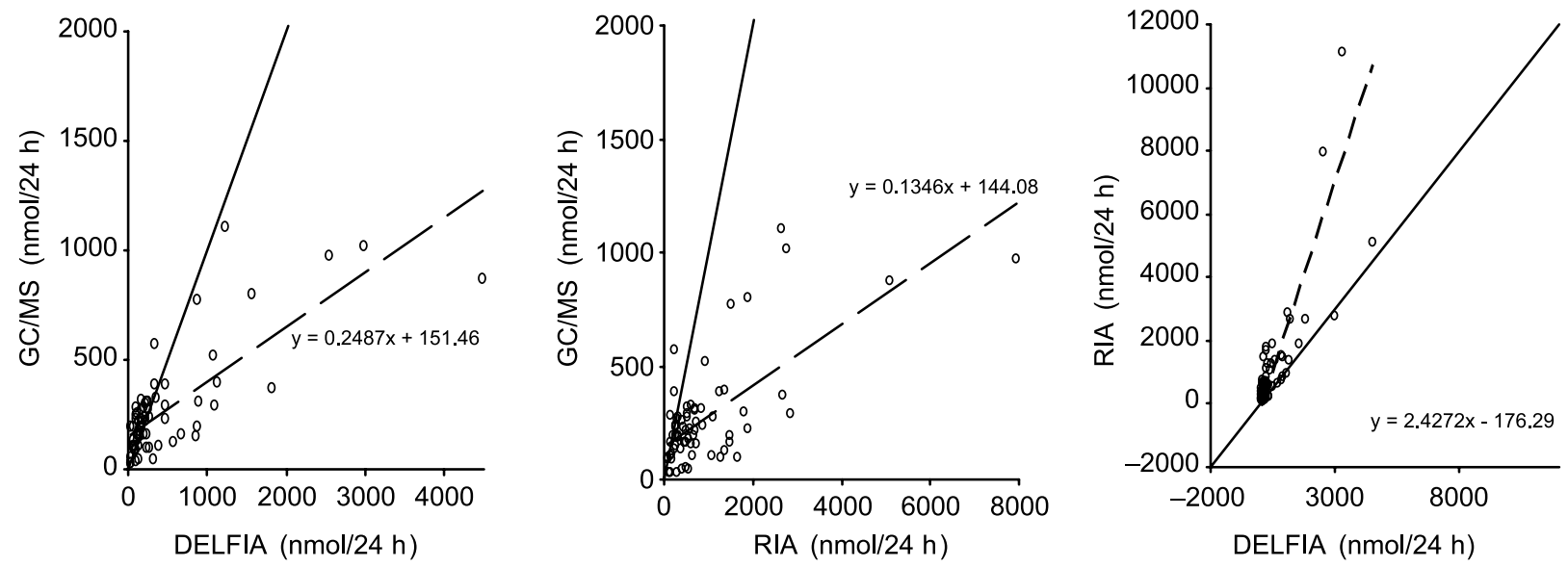

Figure 1 Comparison of urine 18-OHF methods. Solid line, slope; Dashed line, identity line $y=x$.

$P<0.0001$ ), but there were large bias differences: RIA bias was greater than DELFIA which was greater than GC-MS (Fig. 1). GC-MS recorded lower values than both immunoassays.

The range of values of 18 -OHF measured in urine using the three methods are illustrated in Fig. 2 and the differences between urine 18-OHF levels for each assay shown in Table 1.

For adenoma, results higher than the normal range were obtained in $6 / 6,6 / 6$ and $2 / 6$ patients in the RIA, DELFIA and GC-MS assays respectively. Discrimination between adenoma and $\mathrm{BAH}$ was best for the DELFIA method, with no overlap between results for these two groups. Thus using the lowest value measured in the adenoma group for each assay as an arbitrary cut-off to distinguish adenoma from $\mathrm{BAH}$ or essential hypertension, the DELFIA assay would correctly predict adenoma in $100 \%$ of cases. The RIA would correctly identify BAH in $33.3 \%$ of cases and essential hypertension in $84.2 \%$ of cases. GC-MS would correctly identify BAH in $33.3 \%$ of cases and essential hypertension in $27.3 \%$ of cases.

All three methods gave significantly elevated results for the GSH group with values higher than normal range obtained in $9 / 9,8 / 9$ and $7 / 8$ cases of GSH in the RIA, DELFIA and GC-MS assays respectively. Using the highest value measured in the adenoma group for each assay as an arbitrary cut-off to distinguish adenoma from GSH, GSH was correctly predicted in $66.6 \%, 66.6 \%$ and $50 \%$ of cases in the DELFIA, RIA and GC-MS assays respectively. Using the highest value measured in the $\mathrm{BAH}$ and essential hypertension groups to distinguish theses conditions from GSH, GSH was correctly predicted in $88.9 \%$, $66.6 \%$ and $62.5 \%$ of cases in the DELFIA, RIA and GC-MS assays respectively.

There were no significant differences in urine 18-OHF measurements in cases of BAH compared with essential hypertension using any of the assays.

\section{Discussion}

This study confirms that measurement of urine 18-OHF may be a useful additional test in determining the differential diagnosis of primary hyperaldosteronism, but shows that the clinical diagnostic value is method dependent.
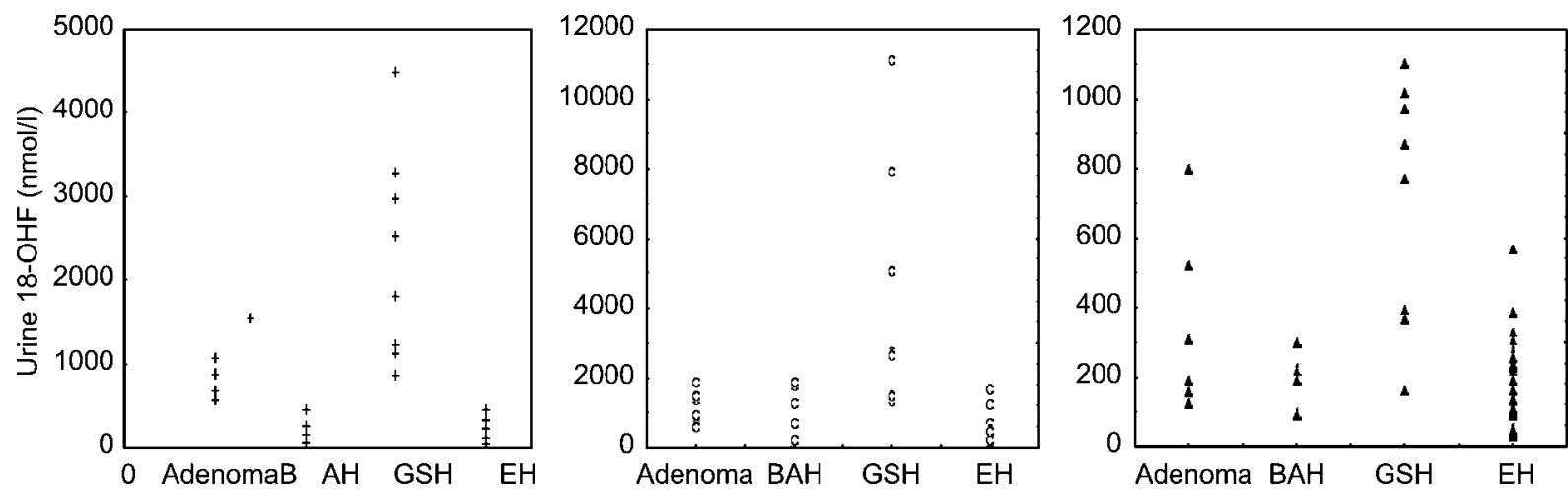

Figure 2 Range of urine 18-OHF levels in adrenal adenoma, bilateral adrenal hyperplasia (BAH), glucocorticoid suppressible hyperaldosteronism (GSH) and essential hypertension (EH) in the three different assays (RIA, RIA and GC-MS). 
Table 1 Comparison of three assays to distinguish urine 18-OHF measurements in patients with adrenal adenoma $(n=6)$, bilateral adrenal hyperplasia (BAH) $(n=6)$, glucocorticoid suppressible hyperaldosteromism (GSH) $(n=6)$ and essential hypertension $(\mathrm{EH})(n=9)$.

\begin{tabular}{lccc}
\hline & RIA & GC-MS & Delfia \\
\hline Adenoma vs BAH & $\mathrm{ns}$ & $\mathrm{ns}$ & 0.002 \\
Adenoma vs GSH & 0.01 & $\mathrm{~ns}$ & $\mathrm{~ns}$ \\
Adenoma vs EH & 0.0005 & $\mathrm{~ns}$ & $<0.0001$ \\
BAH vs GSH & 0.01 & 0.008 & 0.005 \\
BAH vs EH & $\mathrm{ns}$ & $\mathrm{ns}$ & $\mathrm{ns}$ \\
GSH vs EH & $<0.0001$ & 0.0003 & $<0.0001$ \\
\hline
\end{tabular}

Values are 2-tailed $P$-values from Mann-Whitney $\mathrm{U}$ test. ns, not significant.

In accord with previous studies we found that patients with a Conn's adenoma or GSH excreted more urine 18-OHF than patients with $\mathrm{BAH}$ or essential hypertension, although the precision of each assay in distinguishing these conditions differed. The DELFIA was the best assay for distinguishing Conn's adenoma from BAH and essential hypertension. Thus using an arbitrary cut-off of the lowest value measured in the adenoma group for each assay it was possible to distinguish all cases of adenoma from $\mathrm{BAH}$ or essential hypertension using the DELFIA assay. In GSH levels of 18-OHF may be up to 10-fold higher than the upper limit of the normal range (11) and in each of the assays levels were significantly higher for GSH compared with essential hypertension and BAH. Although there was still some overlap when results were compared with adenoma the findings suggest measurement of urine 18-OHF in suspected GSH is a useful test whilst waiting for definitive genotyping. Unlike previous studies, the levels of 18-OHF measured in those patients with BAH were not significantly different from patients with essential hypertension (5). This may be partly due to difficulty in diagnosing $\mathrm{BAH}$, and hence misclassification using available tests. Likewise although high levels of 18-OHF have previously been reported in urine from patients with 'essential hypertension' (12), values may not always differ from normotensive patients (13). Thus measurements of urine 18-OHF may help to exclude Conn's adenoma and GSH but are not otherwise useful in the diagnosis or management of BAH and essential hypertension.

The poor discrimination of the GC-MS method was surprising as the assay was not operating near the detection limit. The original measurements of 18-OHF in urine (3) were by GC-MS, but the technique may not be widely available and the method is time-consuming. GC-MS is generally accepted as the 'gold standard' as it is significantly more specific than immunoassay. The higher results and better discrimination by immunoassay could be explained by the presence of a related, as yet unidentified, steroid that cross-reacts in the immunoassays. However, the specificity of the antiserum used in the DELFIA assay is very high, particularly in terms of cross-reactivity with 18-hydroxycorticosterone and certainly discrimination was not as good using the RIA, in which the antibody used is less specific. However, this possibility merits further study such as using an affinity purifed antibody (13) or a purification step before immunoassay (14). An alternative possibility is that the GC-MS method is not measuring different forms of 18-OHF, but on further testing we have found no evidence for this (M Wallace, personal communication). RIAs have been used in clinical investigation for measuring 18-OHF but may have low clinical applicability and use radioactive reagents that are not readily available. The time-resolved fluoroimmunoassay avoids use of radioactivity and this technique has advantages of higher sensitivity, better reproducibility and shorter development time compared with other methods. In this study the best discrimination of 18-OHF measurements using the DELFIA assay indicates this assay may be suitable for a centralised service.

\section{References}

1 Young WF. Minireview: Primary aldosteronism - changing concepts in diagnosis and treatment. Endocrinology $2003 \mathbf{1 4 4}$ 2208-2213.

2 Stewart PM. Mineralocorticoid hypertension. Lancet 1999353 1341-1347.

3 Ulick S \& Chu MD. Hypersecretion of a new corticosteroid, 18-hydroxycortisol, in two types of adrenocortical hypertension. Clinical and Experimental Hypertension 19824 1771-1777.

4 Chu MD \& Ulick S. Isolation and identification of 18-hydroxycortisol from urine of patients with primary aldosteronism. Journal of Biological Chemistry $19822572218-2224$.

5 Corrie JET, Edwards CE \& Budd PS. A radioimmunoassay for 18-hydroxycortisol in plasma and urine. Clinical Chemistry $198531849-852$.

6 Wenting GJ, Man in't Veld J, Derkx FH, Brummelen PV \& Schalekamp MA. ACTH-dependent aldosterone excess due to adrenocortical adenoma: a variant of primary aldosteronism. Journal of Clinical Endocrinology and Metabolism $1978 \mathbf{4 6}$ 326-335.

7 Reincke M, Beuschlein F, Latronico AC, Arlt W, Chrousos GP \& Allolio B. Expression of adrenocorticotrophic hormone receptor mRNA in human adrenocortical neoplasms: correlation with P450scc expression. Clinical Endocrinology $1997 \mathbf{4 6}$ $619-626$.

8 Lifton RP, Dluhy RG, Powers M, Rich GM, Cook S, Ulick S \& Lalouel JM. A chimeric 11 $\beta$-hydroxylase/aldosterone synthase gene causes glucocorticoid-remediable aldosteronism and human hypertension. Nature $1992355262-265$.

9 Pascoe L, Curnow KM, Slutsker L, Connell JMC, Speiser PW, New MI \& White PC. Glucocorticoid-suppressible hyperaldosteronism results from hybrid genes created by unequal crossovers between CYP11B1 and CYP11B2. PNAS 199289 8327-8331.

10 Shackleton CHL. Mass spectrometry in the diagnosis of steroidrelated disorders in hypertension research. Journal of Steroid Biochemistry and Molecular Biology 198345 1127-1140.

11 Gómez-Sánchez CE, Montgomery M, Ganguly A, Holland OB, Gómez-Sánchez EP, Grim CE \& Weinberger MH. Elevated urinary excretion of 18-oxocortisol in glucocorticoid-suppressible 
aldosteronism. Journal of Clinical Endocrinology and Metabolism $1984591022-1024$

12 Gómez-Sánchez C, Gómez-Sánchez E \& Holand B. Role of 18-hydroxylated cortisols in hypertension. Journal of Steroid Biochemistry 198727 971-975.

13 Mosso L, Gomez-Sanchez CE, Foecking MF \& Fardella C. Serum 18-hydroxycortisol in primary aldosteronism, hypertension, and normotensives. Hypertension $20013 \mathbf{3 8} 688-691$.
14 Stowasser M, Bachmann AW, Tunny TJ \& Gordon RD. Production of 18-oxo-cortisol in subtypes of primary aldosteronism. Clinical and Experimental Pharmacology \& Physiology 199623 591-593.

Received 17 November 2004

Accepted 9 March 2005 